\title{
Discovery of an X-Ray Off-State in the Supersoft Source CAL 83
}

\author{
P. Kahabka \\ Astronomical Institute and Center for High Energy Astrophysics, \\ University of Amsterdam, Kruislaan 403, NL-1098 SJ Amsterdam
}

\begin{abstract}
An X-ray off state of CAL 83 has been observed in April 1996 with the ROSAT HRI three weeks after a normal on state. An upper limit for a linear decline time of the observed flux of $\sim 20$ days (and of an efolding decline time of $\sim 6$ days) is deduced. This decline may be due to the response of the white dwarf envelope to a temporary increased mass accretion rate giving rise to an envelope expansion. CAL 83 may resemble the recurrent supersoft LMC transient RX J0513.9-6951 with episodes of disappearance in X-rays.
\end{abstract}

\section{Introduction}

CAL 83 can be considered as the prototype supersoft $\mathrm{X}$-ray source. It was discovered with the Einstein satellite (Long et al. 1981) and identified in the optical with a blue emission line star in a binary system with an orbital period of 1.04 days.

\section{Discussion and conclusions}

CAL 83 has been considered as a persistent supersoft source as it has never been reported to be observed during an X-ray off state. ROSAT observations did not reveal strong variability in X-rays. The variability found in Einstain data (Brown et al. 1994) may be due to instrumental effects (E. Gotthelf, private communication). The source has been found to be variable in the UV (Gänsicke et al. 1996) and in the optical (Crampton et al. 1996). A recent $\mathrm{X}$-ray off state has been discovered from this source which turned out to be of temporary nature (cf. Figure 1, Kahabka 1996b,c). CAL 83 may resemble the recurrent supersoft LMC transient RX J0513.9-6951 which turns off in X-rays every 100-200 days. In this transient it is believed that mass accretion rates close to $10^{-6} \mathrm{M}_{\odot} \mathrm{yr}^{-1}$ (Southwell et al. 1996) lead occasionally to a bloated white dwarf with an extended envelope so large that it avoids the detection of the source in X-rays during these periods. The mechanism behind this recurrent blow-up of the white dwarf envelope may be due to recurrent nuclear burning on a massive $\left(\mathrm{M}_{\mathrm{WD}} \sim 1.3 \mathrm{M}_{\odot}\right.$ ) white dwarf (Kahabka 1996a) or due to episodic mass-transfer from the donor star, caused by a mechanism like magnetic star spots crossing episodically the L1 point (cf. Southwell et al. 1996). The short e-folding decline time for the X-ray turn-off of CAL 83 of $<6$ days) allows to constrain the underlying physical mechanism. If it is due to the response of the white dwarf envelope to a temporary increase of the mass accretion rate, then it may be determined by the envelope expansion time. An X-ray decline (by a 
factor of 50 ) within $\sim 66$ days has been observed in the symbiotic star AG Dra which is supposed to contain a lower mass white dwarf $\left(\mathrm{M}_{\mathrm{WD}} \sim 0.53 \mathrm{M}_{\odot}\right.$, Mikolajewska \& Keyon 1992). The X-ray decline is supposed to be due to an expanding white dwarf envelope responding to an increase in the mass transfer rate onto the white dwarf by a factor of $\sim 30 \%$ (Greiner et al. 1996).

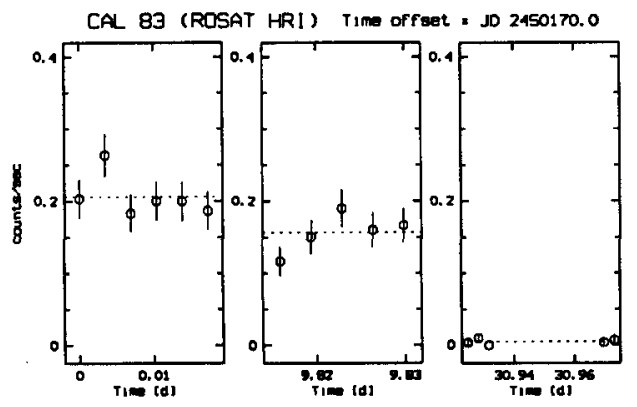

Figure 1. X-ray light curve of CAL 83. Data points and the mean count rate (dashed line) are given. Time offset is JD 2450170.0 (27.5 Mar 1996).

Acknowledgments. I thank Mario Livio for comments. P.K. is a Human Capital and Mobility fellow.

\section{References}

Brown, T., Cordova, F., Ciardullo, R., et al. 1994, ApJ, 422, 118

Crampton D., Hutchings B., Cowley A.P., et al., 1996, ApJ 456, 320

Gänsicke, B.T., Beuermann, K., \& de Martino, D. 1996, in: workshop on supersoft sources, ed. J. Greiner, Lecture Notes in Physics, Springer, p.107

Greiner, J., Hasinger, G., \& Kahabka, P. 1991, A\&A, 246, L17

Greiner, J., Bickert, K., Luthardt, R., et al. 1996, UV and X-Ray Monitoring of AG Draconis During the 1994/1995 Outbursts, in: workshop on supersoft sources, ed. J. Greiner, Lecture Notes in Physics, Springer, p.267

Kahabka, P. 1996a, Transient and Recurrent Supersoft Sources as Progenitors of Type Ia Supernovae and Accretion Induced Collapse, in: workshop on supersoft sources, ed. J. Greiner, Lecture Notes in Physics, Springer, p. 215

Kahabka, P. 1996b, IAU Circ. No. 6432

Kahbaka, P. 1996c, Discovery of an X-ray off-state in the supersoft source CAL 83, A\&AL, (subm.)

Long, K.S., Helfand, D.J., \& Grabelsky, D.A. 1981, ApJ, 248, 925

Mikolajewska, J., \& Kenyon, S.J. 1992, MNRAS, 256, 177

Southwell, K.A., Livio, M., Charles, P.A., et al. 1996, ApJ, (in press) 